\title{
Incidental alpha-1-antitrypsin deficiency found in post-transplant liver allografts: Report of two cases
}

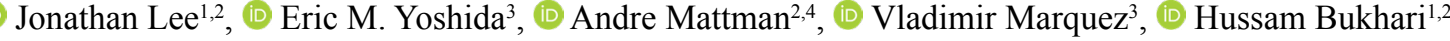 \\ (D) David Farnell ${ }^{1 *}$, (1) Hui-Min Yang ${ }^{1,2 *}$
}

${ }^{I}$ Department of Pathology and Laboratory Medicine, Vancouver General Hospital, Vancouver, BC, Canada; ${ }^{2}$ Department of Pathology and Laboratory Medicine, University of British Columbia, Vancouver, BC, Canada, ${ }^{3}$ Department of Gastroenterology, Vancouver General Hospital, Vancouver, BC, Canada; ${ }^{4}$ Department of Pathology and Laboratory Medicine, St. Paul's Hospital, Vancouver, BC, Canada

\begin{abstract}
Alpha-1 antitrypsin deficiency is an autosomal recessive disease most commonly caused by misfolding of the Alpha-1-antitrypsin protein, which prevents its release from hepatocytes into the systemic circulation. This results in increased lifetime risk of liver and lung disease. Due to its variable penetrance, presentation and natural history, patients with alpha-1 antitrypsin deficiency are often underdiagnosed. In this report, we present two cases of alpha-1 antitrypsin deficiency in deceased-donor liver transplant allografts diagnosed post-transplant. There is currently no known adverse outcome directly linked to alpha-1 antitrypsin deficiency in the immediate post-transplant follow-up period. Thus, these allografts should not be excluded from transplantation.
\end{abstract}

Keywords: Alpha 1-antitrypsin deficiency; case study; liver transplantation.

\section{Introduction}

Alpha-1 antitrypsin deficiency (AATD) has an estimated prevalence of 1: 5,000 to 1: 10,000 in the USA and Canada. ${ }^{[1]}$ Most commonly, AATD is the result of misfolding of the AAT protein within hepatocytes, preventing its release into the systemic circulation. The illness typically presents as early-onset emphysema secondary to a lack of AAT-mediated inhibition of neutrophil elastase, which leads to damage to pulmonary connective tissue. ${ }^{[1]}$ Hepatotoxicity arises from the accumulation of misfolded AAT protein, which polymerizes within the endoplasmic

\section{*: Both authors contributed equally to this work.}

How to cite this article: Lee J, Yoshida EM, Mattman A, Marquez V, Bukhari $H$, Farnell D, Yang HM. Incidental alpha-1-antitrypsin deficiency found in post-transplant liver allografts: Report of two cases. Hepatology Forum 2021; 2(1):31-33.

Received: June 25, 2020; Accepted: August 27, 2020; Available online: January 08, 2021

Corresponding author: Jonathan Lee; Department of Pathology and Laboratory Medicine, Vancouver General Hospital, 910 West $10^{\text {th }}$ Avenue Vancouver, BC, Canada, V5Z $1 \mathrm{M} 9$

Phone: +1-780-719-1489; e-mail: Jlee20@alumni.ubc.ca reticulum of hepatocytes. ${ }^{[2]}$ These AAT globules are visible to light microscopy as hepatocellular inclusions that are digested periodic acid-Schiff (PASD)-positive. These AAT inclusions can be distinguished from other PASD-positive globules by immunohistochemical staining with an AAT-specific antibody. Point mutation of the SERPINA1 gene is the underlying genetic determinant of AATD, with individuals homozygous for the $\mathrm{Z}$ mutation ( $\mathrm{ZZ}$ ) being at risk of developing severe lung and/or liver disease, and in exceedingly rare cases, panniculitis or systemic vasculitis. ${ }^{[1,3]}$ The penetrance of the disease-causing $\mathrm{ZZ}$ gene is variable, with other genetic and environmental factors influencing disease progression. ${ }^{[1]}$ Important predisposing factors include viral hepatitis and alcohol abuse in the case of AATD-associated liver disease and smoking in AATD-associated emphysema. ${ }^{[1]}$

Individuals heterozygous for the $\mathrm{Z}$ mutation (MZ) are generally considered asymptomatic carriers; however, an overrepresentation of $\mathrm{MZ}$ patients has been reported in retrospective studies of chronic liver disease cohorts. ${ }^{[2]}$ A mutant $\mathrm{S}$ allele has also shown a rare association with AATD in individuals heterozygous with a $\mathrm{Z}$ allele (SZ); however, this association is not demonstrated in homozygous (SS) individuals or in heterozygous individuals with a normal allele (MS). ${ }^{[2]}$

We present two cases of AATD discovered incidentally in deceased-donor liver transplant (DDLT) allografts in the post-transplant period. While it is a rare phenomenon with only two previous reports from deceased donors, ${ }^{[4,5]}$ the finding of AATD in the post-transplant setting raises the question of whether AATD poses any significant detriment to the longevity of a DDLT allograft.

\section{Case Report}

Both patients included in this case series were DDLT recipients who were followed by the Solid Organ Transplant Clinic at the Vancouver General Hospital in Vancouver, Canada, under the care of transplant hepatologists for ongoing post-liver transplant follow-up. Histologic analysis of tissue specimens from the liver allografts were performed at the Vancouver General Hospital Department of Pathology and Laboratory Medicine by a gastrointestinal-liver subspecialty pathologist. Molecular analysis of whole blood and liver tissue specimens were performed at St. Paul's Hospital Department of Pathology and Laboratory Medicine, medical biochemistry division in Vancouver.

Written informed consent was obtained from both patients for the use of their clinical information in accordance with the Declaration of Helsinki. The health authority in the province of British Columbia has a policy 


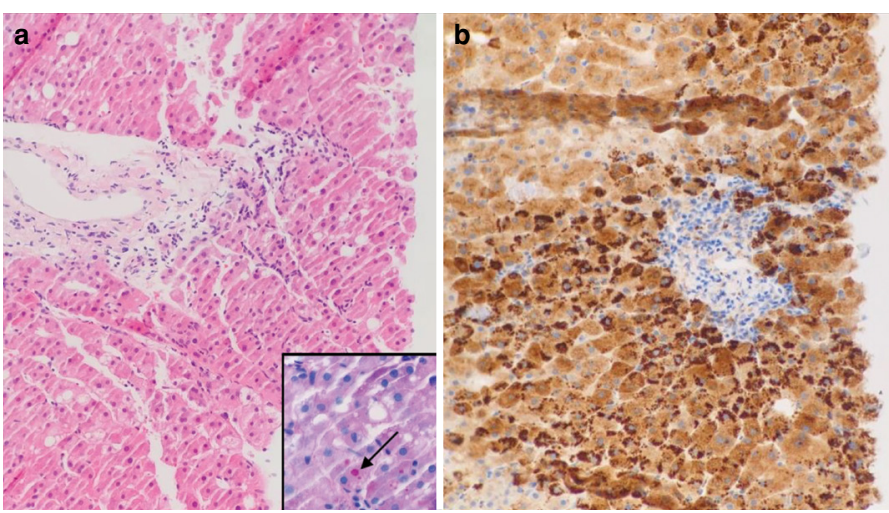

Figure 1. Case one, core needle biopsies from allograft liver. (a) H\&E staining at 20x showing mild steatohepatitis with mild duct injury. Inset: PASD stain at 40x demonstrating eosinophilic intracytoplasmic inclusions representing AAT globules (arrow). (b) AAT immunohistochemical stain confirming AAT globules.

of anonymity for organ donors. Therefore, it was not possible to obtain clinical or demographic information about the donors.

Standard procedure for DDLT at the Vancouver General Hospital includes a screening of potential donors for malignancy, active bacterial infection, HIV, as well as hepatitis B and C. The transplant surgeons visually inspect the donor allograft to assess the degree of steatosis, obtaining a frozen microscopic section for confirmation if it is of borderline appearance. No ancillary testing is performed in a DDLT setting due to the time constraints for allograft viability.

Our first patient is a 62-year old male who underwent DDLT six years previously for end-stage liver disease secondary to chronic hepatitis $\mathrm{C}$ (genotype 3a) and hepatocellular carcinoma. His past medical history is remarkable for hypertension and chronic obstructive pulmonary disease secondary to a 36-pack year smoking history. Post-liver transplant, he continues to smoke 10 cigarettes daily and drinks 3 oz of spirits daily.

The patient's post-transplant liver biochemistry was stable in the first year but became increasingly abnormal by year two. This was believed to be secondary to recurrent hepatitis $\mathrm{C}$, and he was subsequently treated using sofosbuvir plus ribavirin, achieving sustained virologic response. However, his liver function test (LFT) remained persistently abnormal on follow-up testing for the following four years. LFT ranges during this period include an AST of 48-64 U/L (ref. 10-38 U/L), ALT 35-44 U/L (ref. 10-55 U/L), GGT 104-121 (ref. 15-80 U/L), alkaline phosphatase 139-150 U/L (ref. 30-135 U/L), and total bilirubin 13-17 $\mu \mathrm{mol} / \mathrm{L}$ (ref. $<20 \mu \mathrm{mol} / \mathrm{L}$ ). Although this low-level cholestatic profile was attributed to his continued alcohol use, further investigation was nonetheless conducted. An MCRP demonstrated mild dilation of the left intrahepatic duct and common bile duct with no discrete obstruction. Assessment of liver fibrosis by transient elastography (FibroScan ${ }^{\circledR}$ ) yielded a liver stiffness of $15.2 \mathrm{kPa}$, indicating the presence of severe fibrosis. ${ }^{[6]} \mathrm{An}$ ensuing core needle liver biopsy (Fig. 1a) demonstrated the following:

1) Mild macrovesicular steatosis (approximately $15 \%$ ) with minimally active steatohepatitis and pericellular, periportal and slender bridging fibrosis.

2) Mild duct injury, likely indicative of prior rejection episode(s), with no significant acute T-cell mediated rejection infiltrate.

3) Periportal AAT inclusions (confirmed using immunohistochemistry; Fig. 1b).

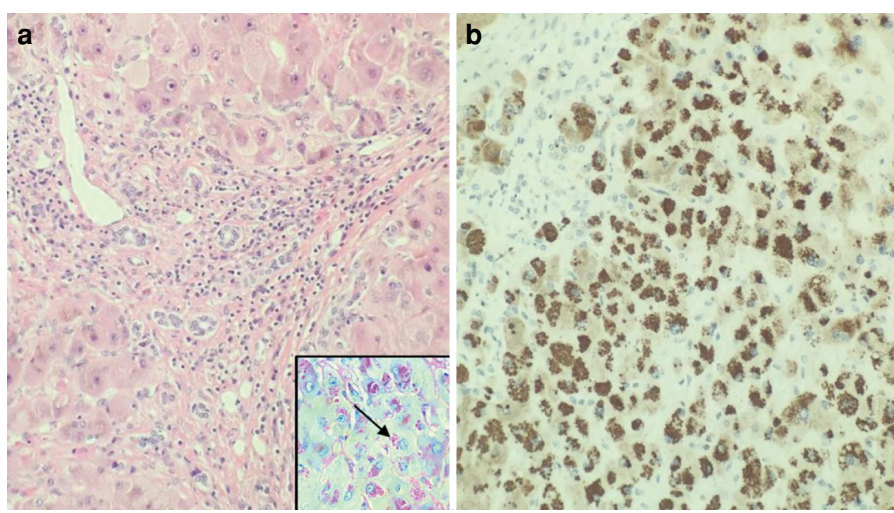

Figure 2. Case two, representative sections of explant liver. (a) H\&E staining at 20x showing chronic rejection, ductopenia, and cirrhosis. Inset: PASD stain at $40 x$ demonstrating eosinophilic intracytoplasmic inclusions representing AAT globules (arrow). (b) AAT immunohistochemical stain confirming AAT globules.

Serum AAT level six months before the liver biopsy was $1.29 \mathrm{~g} / \mathrm{L}$, falling to $1.17 \mathrm{~g} / \mathrm{L}$ on repeat testing (reference range $0.90-2.00 \mathrm{~g} / \mathrm{L}$ ). As expected, genetic testing performed on the patient's whole blood confirmed the patient's genotype as MM, while AAT genotype testing of the allograft biopsy revealed an AATD carrier phenotype (MZ).

Our second patient is a 67 -year old woman who received a liver transplant 23 years ago for acute fulminant liver failure secondary to autoimmune hepatitis. She underwent a repeat liver transplant four years ago, performed due to decompensation with ascites and increasing hepatic encephalopathy. This was preceded by a 2-year history of a nonspecific, gradual increase in alkaline phosphatase and GGT, with recent evidence of chronic rejection on liver biopsy. Lab value ranges during this period include an AST of 59-72 U/L (ref. 10-38 U/L), ALT 20-28 U/L (ref. 10-55 U/L), GGT 32-132 (ref. 15-80 U/L), alkaline phosphatase 160-209 U/L (ref. 30-135 U/L), and total bilirubin 11-153 $\mu \mathrm{mol} / \mathrm{L}$ (ref. $<20 \mu \mathrm{mol} / \mathrm{L}$ ). She is a non-smoker and does not drink alcohol.

On histologic analysis, the explanted allograft specimen demonstrated chronic rejection with ductopenia and cirrhosis (Fig. 2a). PASD staining performed on a representative block showed PASD-positive intracytoplasmic globules, which were confirmed to be AAT inclusions on subsequent AAT immunohistochemistry (Fig. 2b). AAT genotype testing of the explanted liver tissue revealed an AATD carrier phenotype (MZ).

\section{Discussion}

Our cases represent two of only six documented cases of AATD detected in a DDLT allograft. ${ }^{[4,5]}$ Of these other four cases, two patients acquired donor ZZ phenotype livers; the other two patients received livers with heterozygous phenotypes (MS and MZ, respectively). In these reports, it appears that the presence of AATD did not adversely affect patient outcomes for the duration of follow-up. ${ }^{[4,5]}$ The case report by Pungpapong et al. ${ }^{[5]}$ describes the post-transplant MS and MZ phenotype patients as having stable graft function at follow-up four years post-transplant. ${ }^{[5]}$ The single ZZ phenotype patient died at 14 years post-transplant of a non-liver related cause with stable graft function. ${ }^{[5]}$ The remaining case reported by Arnal et al.$^{[4]}$ of an adult male patient receiving a ZZ phenotype liver describes the patient as remaining asymptomatic at six years post-transplant, with only slightly elevated cholestatic enzymes and a repeat liver biopsy finding of mild chronic portal hepatitis with portal fibrosis and multiple PASD-positive globules. ${ }^{[4]}$ 
The indolent course of AATD in post-liver transplant patients is consistent with the natural history of the disease, with liver function typically preserved for many years in the absence of additional hepatic injury. ${ }^{[2]}$ It is also supported by preliminary evidence showing no significant difference in clinical outcomes in live-donor liver transplants from AATD-heterozygous donors compared to transplants from normal living donors within a short (3 months) follow-up period. ${ }^{[7]}$ DDLT livers are not routinely screened for AATD. Its presence does not preclude using the organ in transplant per se if otherwise healthy. [7] Hypothetically speaking, the first patient in our series may be at increased risk of emphysema and progressive liver damage in the context of an MZ allograft phenotype, given his continued smoking and alcohol use. Smoking and alcohol cessation will be an important consideration for him, as will be the need for close clinical follow-up to monitor for disease progression.

Our overall experience suggests that liver allografts from donors with AATD of the MZ genotype may be used successfully, at least in the medium term. An interesting implication of this finding is that it raises the possibility of using livers from patients with AATD in "domino" transplants, which are currently performed in conditions such as Familial Amyloidotic Polyneuropathy. ${ }^{[8]}$ For instance, patients with AATD undergoing liver transplantation could potentially become donors for patients with acute liver failure needing urgent transplantation since AATD liver disease tends to progress relatively slowly. We believe this would be feasible and should be the subject of further research.

Acknowledgements: We thank Dr. Gordon Ritchie, clinical molecular scientist, and Ms. Laura Burns, technical coordinator, at St. Paul's Hospital for their technical expertise and help in specimen molecular genotyping.
Informed Consent: Written informed consent was obtained from the patients for the publication of the case report and the accompanying images.

Peer-review: Externally peer-reviewed.

Author Contributions: All authors contributed to the concept and design, data acquisition, and/or writing of the article.

Conflict of Interest: The authors have no conflict of interest to declare.

Financial Disclosure: The authors declared that this study has received no financial support.

\section{References}

1. De Serres F, Blanco I. Role of alpha-1 antitrypsin in human health and disease. J Intern Med. 2014 Oct;276(4):311-35.

2. Patel D, Teckman JH. Alpha-1-Antitrypsin Deficiency Liver Disease. Clin Liver Dis. 2018 11;22(4):643-55.

3. Tanash HA, Piitulainen E. Liver disease in adults with severe alpha-1-antitrypsin deficiency. J Gastroenterol. 2019 Jun;54(6):541-8.

4. Arnal FM, Lorenzo MJ, Suárez F, Otero A, Alonso C, Filgueira P, et al. Acquired PiZZ alpha-1 antitrypsin deficiency in a liver transplant recipient. Transplantation. 2004 Jun 27;77(12):1918-9.

5. Pungpapong S, Krishna M, Abraham SC, Keaveny AP, Dickson RC, Nakhleh RE. Clinicopathologic findings and outcomes of liver transplantation using grafts from donors with unrecognized and unusual diseases. Liver Transpl. 2006 Feb;12(2):310-5.

6. De Lédinghen V, Vergniol J. Transient elastography (FibroScan). Gastroenterol Clin Biol. 2008 Sep;32(6 Suppl 1):58-67.

7. Doshi SD, Wood L, Abt PL, Olthoff KM, Shaked A, Goldberg DS, et al. Outcomes of Living-donor Liver Transplantation Using Grafts Heterozygous for a-1 Antitrypsin Gene Mutations. Transplantation. 2019 06;103(6):1175-80.

8. Popescu I, Dima SO. Domino liver transplantation: how far can we push the paradigm? Liver Transpl. 2012;18(1):22-8. 